\title{
Charge frustration and quantum criticality for strongly correlated fermions
}

\author{
Liza Huijse, ${ }^{1}$ James Halverson, ${ }^{2}$ Paul Fendley, ${ }^{2}$ and Kareljan Schoutens ${ }^{1}$ \\ ${ }^{1}$ Institute for Theoretical Physics, University of Amsterdam, \\ Valckenierstraat 65, 1018 XE Amsterdam, The Netherlands \\ ${ }^{2}$ Department of Physics, University of Virginia, Charlottesville, VA 22904-4714 USA
}

(Dated: April 1, 2008, revised: October 08, 2008)

\begin{abstract}
We study a model of strongly correlated electrons on the square lattice which exhibits charge frustration and quantum critical behavior. The potential is tuned to make the interactions supersymmetric. We establish a rigorous mathematical result which relates quantum ground states to certain tiling configurations on the square lattice. For periodic boundary conditions this relation implies that the number of ground states grows exponentially with the linear dimensions of the system. We present substantial analytic and numerical evidence that for open boundary conditions the system has gapless edge modes.
\end{abstract}

Quantum criticality is a key notion in the analysis of non-Fermi liquid behavior of strongly correlated electrons. In general, quantum criticality is expected when parameters are tuned such that competing orders are perfectly balanced [1]. In two or three spatial dimensions, the identification of quantum critical behavior is quite difficult, due to a lack of sufficiently powerful analytic methods and to the limited reach of numerics.

In this Letter, we identify quantum critical behavior in a specific model for spinless itinerant fermions on a twodimensional square lattice. The Hamiltonian combines standard hopping terms with strong repulsive interactions. At filling $f=1 / 2$ (one fermion per two sites) this model develops a Mott insulating phase with a fermion on every other lattice site. In [2], it was shown that a related model exhibits a stripe phase upon modest doping. Here we study the model in the 'strongly doped Mott insulator phase' at intermediate density.

In the model at hand, interactions have been tuned to obtain a property called supersymmetry, which we employed in our analysis. In previous work (for a review see [3]), we demonstrated that this model develops a form of quantum charge frustration that leads to an extensive ground state entropy [4, [5]. Here we show that this same model on the square lattice with an edge possesses quantum critical edge excitations. We find that this quantum criticality arises due to the competition of two forms of charge order at densities $f=1 / 5$ and $f=1 / 4$.

Our degrees of freedom are spinless fermions living on the square lattice. A fermion at site $i$ is created by the operator $c_{i}^{\dagger}$ with $\left\{c_{i}, c_{j}^{\dagger}\right\}=\delta_{i j}$. The fermions have a hard core, meaning that they are not only forbidden to be on the same site as required by Fermi statistics, but are also forbidden to be on adjacent sites. Their creation operator is $d_{i}^{\dagger}=c_{i}^{\dagger} \mathcal{P}_{<i>}$, where

$$
\mathcal{P}_{<i>}=\prod_{j \text { next to } i}\left(1-c_{j}^{\dagger} c_{j}\right)
$$

is zero if any site next to $i$ is occupied.

Our model, first introduced in [6], is easiest to define in terms of the "supersymmetry" operator $Q=\sum_{i} d_{i}^{\dagger}$.
The Hamiltonian is

$$
H=\left\{Q, Q^{\dagger}\right\}=\sum_{<i j>} d_{i}^{\dagger} d_{j}+\sum_{i} \mathcal{P}_{<i>} .
$$

The model is supersymmetric because $Q^{2}=\left(Q^{\dagger}\right)^{2}=0$, which then implies that $[Q, H]=\left[Q^{\dagger}, H\right]=0$. The latter term in the Hamiltonian combines a chemical potential and a repulsive next-nearest-neighbor potential. On the square lattice it reads

$$
\sum_{i} \mathcal{P}_{<i>}=N-4 F+\sum_{i} V_{<i>}
$$

where $V_{<i>}+1$ is the number of particles adjacent to $i$, unless there are none, in which case $V_{<i>}=0 . N$ is the number of sites. The operator $F=\sum_{i} d_{i}^{\dagger} d_{i}$ counts the number of fermions and commutes with the Hamiltonian. The chemical potential is fixed at $\mu=4$ and there is no a priori constraint on the particle number.

The problem of counting the ground states in this supersymmetric lattice model turns out to be related to some simple-to-describe (but often difficult to solve) geometrical counting problems (see [4, 6]). A heuristic way of understanding this is from the "3-rule": to minimize the energy, fermions prefer to be mostly 3 sites apart (with details depending on the lattice). Using techniques from cohomology, it was proved for several lattices that the configurations which satisfy the 3-rule are in one-toone correspondence with ground states [4].

The correspondence between these configurations and ground states holds for a wide variety of boundary conditions. This indicates that the ground state wavefunctions exhibit a form of charge ordering. This ordering is akin to the Néel ordering in an antiferromagnet: the ordered state is not an exact eigenstate, but one can find a non-vanishing antiferromagnetic order parameter for the ground state. In this sense, the ordered state dominates the ground state. For our model, the structure is even richer, because there are typically an exponentially growing number of ways of satisfying the 3-rule. Such behavior is what we mean by charge frustration. On 
generic lattices, the number of ground states grows exponentially with the full (two-dimensional) volume of the system [5, 6], see also [7]. However, for the square lattice described here, we will show that for periodic boundary conditions the number of ground states grows exponentially with the linear dimensions of the system.

An important object in supersymmetric theories is the Witten index $W$, which is defined as $W=N_{b}-N_{f}$, where $N_{b}$ is the number of bosonic ground states (those with an even number of fermions), and $N_{f}$ is the number of fermionic ground states (those with an odd number of fermions) [8]. By definition, $W$ is a lower bound on the number of ground states.
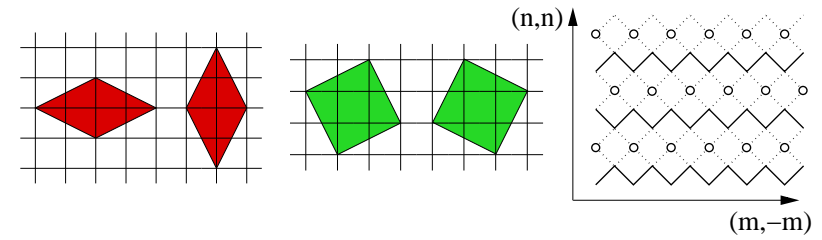

FIG. 1: The diamonds on the left and squares in the middle. On the right we show the square lattice rotated by $45^{\circ}$. Sublattice $S_{1}$ is indicated by the circles and sublattice $S_{2}$ is indicated by the drawn lines.

We show in this paper that for the square lattice, the ground states are in one-to-one correspondence with configurations that come from tiling the plane using the four rhombuses illustrated in fig. 1, which for obvious reasons we call diamonds and squares. Here the particles sit at the corners of the rhombuses, along the edges they are 3 sites apart. A key result was proved rigorously by Jonsson [9]. He showed that for the square lattice with periodic boundary conditions in both directions, the Witten index is simply related to tilings with these rhombuses. Precisely, let $t_{b}\left(t_{f}\right)$ be the number of ways of tiling the torus with these four rhombuses, so that there are an even (odd) number of fermions [or equivalently, an even (odd) number of rhombuses]. The most general version of this theorem allows for any type of torus on the square lattice with periodicities $\vec{u}=\left(u_{1}, u_{2}\right)$ and $\vec{v}=\left(v_{1}, v_{2}\right)$. The expression for the Witten index then reads [9]

$$
W_{u, v}=N_{b}-N_{f}=t_{b}-t_{f}-(-1)^{d_{-}} \theta_{d_{-}} \theta_{d_{+}},
$$

where $d_{ \pm} \equiv \operatorname{gcd}\left(u_{1} \pm u_{2}, v_{1} \pm v_{2}\right)$ and

$$
\theta_{d} \equiv \begin{cases}2 & \text { if } d=3 k, \text { with } k \text { integer } \\ -1 & \text { otherwise. }\end{cases}
$$

A natural extension of Jonsson's theorem is to relate the total number of ground states to rhombus tilings:

$$
N_{b}+N_{f}=t_{b}+t_{f}+\Delta,
$$

where $|\Delta|=\left|\theta_{d_{-}} \theta_{d_{+}}\right|$. When $\vec{u}=(m,-m)$ and $v_{1}+v_{2}=3 p$ we prove this relation explicitly with $\Delta=-(-1)^{\left(\theta_{m}+1\right) p} \theta_{d_{-}} \theta_{d_{+}}$(see below).
In our supersymmetric models, the problem of computing the total number of ground states reduces to finding the cohomology of the supercharge $Q[\underline{6}]$. More precisely, the dimension of the non-trivial cohomology of $Q$ corresponds to the total number of linearly independent ground states. The cohomology is the vector space of states which are annihilated by $Q$ but which are not $Q$ of something else.

To compute the cohomology is in general very difficult. A useful theorem is the 'tic-tac-toe' lemma of [10]. This says that under certain conditions, the cohomology $H_{Q}$ for $Q=Q_{1}+Q_{2}$ is the same as the cohomology of $Q_{1}$ acting on the cohomology of $Q_{2}$. In an equation, $H_{Q}=$ $H_{Q_{1}}\left(H_{Q_{2}}\right) \equiv H_{12}$, where $Q_{1}$ and $Q_{2}$ act on different sublattices $S_{1}$ and $S_{2}$.

The crucial step is to choose the right sublattices: for the square lattice we take a set of disconnected sites for $S_{1}$ and a set of (periodic) chains for $S_{2}$ (see fig. 1). For free boundary conditions in either one or both of the diagonal directions $[(m,-m)$ and $(n, n)]$ the full cohomology problem has been solved using Morse theory [11]. These cases can also be solved using 'tic-tac-toe' with $S_{1}$ and $S_{2}$ as in fig. 11 To solve $H_{Q_{2}}$, we start from the bottom chain. If a site on $S_{1}$ directly above this chain is occupied, there is an isolated site on the bottom chain, leading to a vanishing $H_{Q_{2}}$. It follows that all sites directly above the bottom chain must be empty. Continuing this argument for subsequent chains, one finds that all sites on $S_{1}$ must be empty. Computing $H_{12}$ is now a trivial step. The dimension of $H_{Q}$ is related to the number of ground states on the chains that constitute $S_{2}$. This depends on the exact boundary conditions. One finds that the dimension of $H_{Q}$ is either one or zero, except for the cylindrical case periodic in the $(m,-m)$-direction with $m=3 p$ and $n=3 q$ or $n=3 q+1$. In that case the dimension of $H_{Q}$ is $2^{K}$, with $K$ the nearest integer to $2 n / 3$.

For periodic boundary conditions in both diagonal directions, solving $H_{Q}$ becomes much more involved. This is due to the fact that in this case solving $H_{Q_{2}}$ does not imply that all sites on $S_{1}$ are empty. Instead, there are many allowed configurations on $S_{1}$, and solving $H_{12}$ becomes highly non-trivial. We tackled this problem in two steps. First, we solved $H_{12}$ for the case where $S_{2}$ consists of 1 chain of arbitrary length, that is $\vec{u}=(m,-m)$ and $\vec{v}=(1,2)$. Then, we extended this to the case where $S_{2}$ consists of an arbitrary number of chains, that is $v_{1}+v_{2}=3 p$. This establishes (10) with $\Delta$ as given above. Our proof, which will be presented elsewhere, resolves the ground state counting problem of this highly frustrated system on the 2D square lattice. Counting rhombus tilings, which is relatively easy [9], shows that indeed the number of ground states grows exponentially with the linear dimensions of the system.

For $\vec{v}=(1,2)$, the tilings reduce to a linear sequence of tiles in arbitrary order. There are zero-energy ground states at all rational filling fractions in the range between 
$1 / 5$ and $1 / 4$. For example, for a system with 30 sites there are 5 linearly independent ground states with 6 particles and 92 with 7 particles. The tilings not only count, but indeed seem to dominate the actual ground states. For specific lengths of the $S_{2}$ chain, we have seen numerically that the charge distribution of a ground state largely overlaps with that of the corresponding tiling. We can exploit this to gain physical insight. There are two uniform phases: all squares at $1 / 5$ filling and all diamonds at $1 / 4$ filling. One diamond in a phase with all squares is then a zero-energy defect with fractional charge $1 / 5$. From counting the number of tilings with one such defect it follows that a defect can have any momentum. This is reminiscent of a flat band. Flat bands usually arise either from tuning the hopping terms of non-interacting particles on an exotic lattice, or from tuning potential terms for strongly interacting particles with negligible kinetic energy [12]. Here, however, the flat band arises from tuning the potentials for particles with kinetic and potential energies of comparable size. Note that filling this flat band with defects is slightly subtle. Since defects cannot sit on top of each other and span over 4 sites, they obey a certain exclusion statistics.

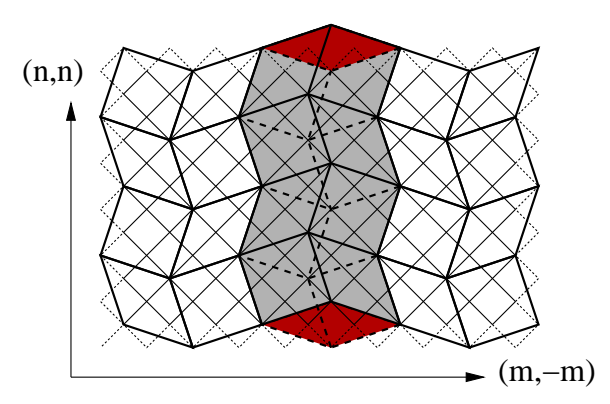

FIG. 2: Edge modes.

We can exploit the effective geometric description of the space of ground states even more by comparing periodic and free boundary conditions. For the square lattice wrapped around the torus with $\vec{u}=(m,-m)$ and $\vec{v}=(n, n)$ the number of ground states grows as $2^{2(n+m) / 3}[9]$. On the cylinder, however, if one cuts the torus open along the $\vec{u}$-direction only $2^{2 n / 3}$ ground states remain. Finally, if one also cuts the cylinder open along the $\vec{v}$-direction one is left with a unique ground state on the plane. What happens to this vast number of ground states that disappear upon changing the boundary conditions? Consider the picture in fig. 2] If one identifies the dotted, zigzagged boundaries both in the $\vec{u}$ as well as in the $\vec{v}$-direction, one finds that both the tiling with the drawn lines as well as the one with the dashed lines represent ground states. However, if one only identifies the boundaries in the $\vec{u}$-direction, then the tiling with the drawn lines no longer represents a ground state. Instead, it has two defects at the edges, which can propagate along the edge. The only available scale for the energy of the edge mode is one over the length of the edge, which suggests that the edge modes are gapless.

To further investigate criticality in this system, we have studied the model numerically with periodic boundary conditions such that $\vec{u}=(L, 0)$ and $\vec{v}$ equals $(0,2)$, $(1,2)$ or $(3,3)$. In all three cases, we have found compelling evidence for critical modes. We investigated how the spectrum changes upon twisting the boundary condition along the $(L, 0)$-direction from periodic to antiperiodic. This is a powerful way of distinguishing between critical and gapped states: for a gapped state, the correlation length is finite and a change in the boundary conditions will have an exponentially small effect on the energy. In contrast, for a critical state the change in the energy will be substantial since the correlation length goes to infinity. More specifically, we will see that the energy has a parabolic dependence on the boundary twist.

In addition to this, one can extract more quantitative properties from twisting the boundary condition. For a critical supersymmetric system in 1D with a Fermi surface, we expect that its continuum limit is described by an $\mathcal{N}=2$ superconformal field theory (SCFT). In such a theory, twisting the boundary condition corresponds to going from the Ramond to the Neveu-Schwarz sector. The twist can be carried out continuously and leads to a spectral flow 13. If we define the twist parameter $\alpha$ to be integer in the Ramond sector and half-integer in the NS sector, the energy is a parabolic function of $\alpha$,

$$
E_{\alpha}=E_{\alpha=0}-\alpha Q_{\alpha=0}+\alpha^{2} c / 3
$$

where $c$ is the central charge. $Q_{\alpha}$ depends linearly on $\alpha$ and is the sum of the left- and right-moving $\mathrm{U}(1)$ charges 13, 14]. Their difference is conserved under the twist and is related to the fermion number. In the lattice model, we can go from periodic to anti-periodic boundary conditions continuously by replacing the term that hops a particle over the boundary $c_{N}^{\dagger} c_{1}+$ h.c. by $e^{2 \pi \imath \alpha} c_{N}^{\dagger} c_{1}+$ h.c. The eigenvalues of the translation operator $p_{\alpha}$ will now depend linearly on the twist parameter:

$$
T_{\alpha}^{L}|\psi\rangle=e^{2 \pi \imath p_{0} L} e^{2 \pi \imath \alpha F}|\psi\rangle \equiv e^{2 \pi \imath p_{\alpha} L}|\psi\rangle,
$$

so $p_{\alpha}=p_{0}+\alpha F / L$ where $L$ is the length of the system and $F$ is the total number of particles in the state $|\psi\rangle$.

We computed the spectrum for various values of the twist parameter via exact diagonalization. We find that the majority of states have a parabolic dependence on the twist parameter by fitting a parabola to the energy levels as a function of the twist parameter, or equivalently, as a function of $p_{\alpha}$. This clearly indicates that the system is critical. An example is shown in fig. 3

For a critical system, the energy of the SCFT in (2) is related to the numerically obtained value of the energy via $E_{\text {num }}=2 \pi E_{\mathrm{CFT}} v_{F} / L$, where $v_{F}$ is the Fermi velocity and $L$ the system size. So by comparing the parabolic 
fit to the numerics with equation (2), we can obtain the ratios $E_{\alpha} / c$ and $Q_{\alpha} / c$.

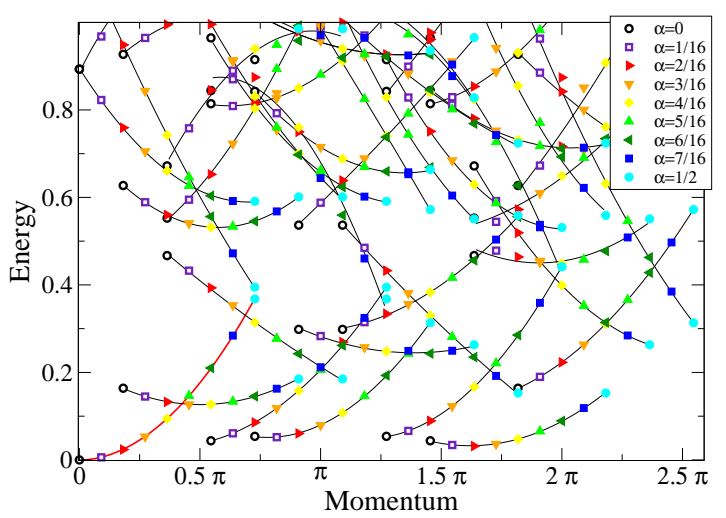

FIG. 3: In the plot we show nine energy spectra as a function of momentum for increasing values of the twist parameter $(0 \leq \alpha \leq 1 / 2$, with steps of $1 / 16)$ for a system with 33 sites $(\vec{u}=(11,0)$ and $\vec{v}=(3,3))$ and 8 fermions. The lines are parabolic fits to the numerical data. The results from the spectral flow analysis for the red line through the origin appear in red in table $\llbracket$

We extracted values for $E_{\alpha} / c$ and $Q_{\alpha} / c$ via the above described method for three models with up to 36 sites (see table \). For the lowest energy levels, we typically find that $(E / c, Q / c)$ in the NS sector is either $(-1 / 12,0)$, $(1 / 12,1 / 3)$ or $(1 / 4,2 / 3)$, all with an accuracy of within $10 \%$. These values occur in the Kac table for the $k$-th minimal model of an $\mathcal{N}=2$ SCFT with $k$ even 14 . This is very compelling evidence that each of these systems is quantum critical.

The fits become less reliable for levels with higher energies, but also if there is an avoided level crossing as a function of the twist. This happens when the energy levels in the Ramond or NS sector are degenerate. For the chain, the avoided crossings vanish in the continuum limit [6] (see also [15]), so one would expect that for the other models this is also merely a finite size feature. For the square ladder $[\vec{u}=(L, 0)$ and $\vec{v}=(0,2)]$, however, the results from exact diagonalization suggest that the avoided crossing will prevail for large system sizes. We are investigating this issue using density matrix renormalization group methods [16].

For the zigzag ladder $[\vec{u}=(L, 0)$ and $\vec{v}=(1,2)$, see fig. 田, we have computed the entanglement entropy of the zero-energy ground states of the system up to 35 sites. We find that for $1 / 4$ and $1 / 5$ filling, the entanglement entropy of the ground states saturates. This implies that the correlation length is finite, and thus the ground states at $1 / 4$ and $1 / 5$ filling are not critical. However, at intermediate fillings we find good correspondence to the spectral flow behavior of an $\mathcal{N}=2$ SCFT. As anticipated in the introduction, this indicates that the system is critical at 2/9 filling, where there is a phase transition due
TABLE I: Results from spectral flow analysis for three types of tori $(L, 0) \times \vec{v}$. Here, $N$ denotes the number of sites and $F$ the number of fermions. We show the results for the lowest energy level for each system. The values for $E$ and $Q$ are given in the NS sector $(\alpha=1 / 2)$ and $c$ is the central charge. For the level in red boldface the results are extracted from the red line in fig. 3

\begin{tabular}{|c|c|c|c|c|}
\hline$N$ & $\vec{v}$ & $F$ & $E / c$ & $Q / c$ \\
\hline 18 & $(3,3)$ & 4 & -0.0851 & 0.004 \\
\hline 36 & $(3,3)$ & 8 & -0.0841 & -0.002 \\
\hline 15 & $(3,3)$ & 4 & 0.0898 & 0.349 \\
\hline 21 & $(3,3)$ & 4 & 0.0850 & 0.337 \\
\hline 24 & $(3,3)$ & 5 & 0.0850 & 0.337 \\
\hline 30 & $(3,3)$ & 7 & 0.0853 & 0.338 \\
\hline 33 & $(3,3)$ & 8 & 0.0855 & 0.338 \\
\hline 9 & $(1,2)$ & 2 & -0.0858 & -0.005 \\
\hline 18 & $(1,2)$ & 4 & -0.0842 & -0.002 \\
\hline 27 & $(1,2)$ & 6 & -0.0839 & -0.001 \\
\hline 17 & $(1,2)$ & 4 & 0.0844 & 0.336 \\
\hline 26 & $(1,2)$ & 6 & 0.0840 & 0.335 \\
\hline 35 & $(1,2)$ & 8 & 0.0839 & 0.335 \\
\hline 14 & $(1,2)$ & 3 & 0.2666 & 0.701 \\
\hline 23 & $(1,2)$ & 5 & 0.2458 & 0.657 \\
\hline 32 & $(1,2)$ & 7 & 0.2432 & 0.652 \\
\hline 16 & $(0,2)$ & 4 & -0.0897 & -0.014 \\
\hline 24 & $(0,2)$ & 6 & -0.0889 & -0.012 \\
\hline 32 & $(0,2)$ & 8 & -0.0885 & -0.011 \\
\hline 12 & $(0,2)$ & 3 & 0.0911 & 0.350 \\
\hline 20 & $(0,2)$ & 5 & 0.0900 & 0.348 \\
\hline 28 & $(0,2)$ & 7 & 0.0894 & 0.347 \\
\hline 14 & $(0,2)$ & 4 & 0.0855 & 0.338 \\
\hline 22 & $(0,2)$ & 6 & 0.0849 & 0.337 \\
\hline 30 & $(0,2)$ & 8 & 0.0847 & 0.336 \\
\hline
\end{tabular}

to competing orders of the diamonds and squares.

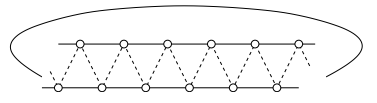

FIG. 4: The figure shows the zigzag ladder $[\vec{u}=(L, 0)$ and $\vec{v}=(1,2)]$, where the zigzagged line is dashed to show that it can also be thought of as a chain with nearest and nextnearest neighbors excluded.

The systems we have investigated numerically are rather confined in one direction. This suggests that we have essentially probed the edge modes and confirmed that they are gapless. Whether there are also gapless modes in the bulk remains unclear from this analysis. However, for a more generic case such as the triangular lattice, where the ground state entropy is truly extensive, one could speculate that a similar argument as above would imply that there are critical modes in the 
bulk as well.

It will be most interesting to investigate whether or not our main findings (quantum charge frustration and quantum criticality induced by strong repulsive interactions for itinerant lattice fermions) carry over to more generic models, and if they can be linked to some of the poorly understood physical features, transport properties in particular, of strange metals and heavy fermions systems displaying non-Fermi liquid behavior.

We thank Pasquale Calabrese for suggesting the entanglement entropy computation. K.S. and L.H. acknowledge financial support through the Research Networking Programme INSTANS of the ESF. P.F. and J.H. were supported in part by NSF grants DMR-0412956 and DMR/MSPA-0704666.

[1] S. Sachdev, Quantum Phase Transitions, (Cambridge University Press, Cambridge, England, 1999).

[2] N.G. Zhang, C.L. Henley, Phys. Rev. B 68, 014506 (2003).

[3] L. Huijse, K. Schoutens, Eur. Phys. J. B 64, 543 (2008).

[4] P. Fendley, K. Schoutens, Phys. Rev. Lett. 95, 046403
(2005)

[5] H. van Eerten, J. Math. Phys. (N.Y.) 46, 123302 (2005).

[6] P. Fendley, K. Schoutens, and J. de Boer, Phys. Rev. Lett. 90, 120402 (2003).

[7] A. Engström, Eur. J. Combinatorics (to be published).

[8] E. Witten, Nucl. Phys. B 202253 (1982).

[9] J. Jonsson, Electronic J. Combinatorics 13(1), \#R67 (2006); Certain Homology Cycles of the Independence Complex of Grid Graphs, preprint (2005); Hard Squares on Grids With Diagonal Boundary Conditions, preprint (2006).

[10] R. Bott, L.W. Tu, Differential Forms in Algebraic Topology, GTM 82, (Springer Verlag, New York, 1982).

[11] M. Bousquet-Melou, S. Linusson, and E. Nevo, J. Alg. Comb. 27, 423-450 (2008).

[12] See e.g. D.L. Bergman, C. Wu, and L. Balents, Phys. Rev. B 78, 125104 (2008); M.E. Zhitomirsky, H. Tsunetsugu, Phys. Rev. B 70, 100403(R) (2004).

[13] A. Schwimmer, N. Seiberg, Phys. Lett. B 184, 191 (1987).

[14] W. Boucher, D. Friedan, and A. Kent, Phys. Lett. B 172, 316 (1986). W. Lerche, C. Vafa, and N. Warner, Nucl. Phys. B 324, 427 (1989).

[15] N. Yu, M. Fowler, Phys. Rev. B 46, 14583 (1992).

[16] L. Huijse, M. Campostrini, and K. Schoutens, in preparation. 